\title{
Which is the best transcranial direct current stimulation protocol for migraine prevention? A systematic review and critical appraisal of randomized controlled trials
}

Raffaele Ornello, Valeria Caponnetto, Susanna Ratti, Giulia D’Aurizio, Chiara Rosignoli, Francesca Pistoia, Michele Ferrara, Simona Sacco (D) and Aurora D'Atri

\begin{abstract}
Background: Transcranial direct current stimulation (tDCS) could counteract the pathophysiological triggers of migraine attacks by modulating cortical excitability. Several pilot randomized controlled trials (RCTs) assessed the efficacy of tDCS for migraine prevention. We reviewed and summarized the state of the art of tDCS protocols for migraine prevention, discussing study results according to the stimulations parameters and patients' populations.

Main body: We combined the keywords 'migraine', 'headache', 'transcranial direct current stimulation', and 'tDCS' and searched Pubmed, Scopus, and Web of Science, from the beginning of indexing to June 22, 2021. We only included RCTs comparing the efficacy of active tDCS with sham tDCS to decrease migraine frequency, intensity, and/or acute drug utilization. The risk of bias of each RCT was assessed by using the RoB-2 tool (Cochrane Collaboration).

Thirteen RCTs (from 2011 to 2021) were included in the review. The included patients ranged from 13 to 135 . RCTs included patients with any migraine $(n=3)$, chronic migraine $(n=6)$, episodic migraine $(n=3)$ or menstrual migraine $(n=1)$. Six RCTs used cathodal and five anodal tDCS, while two RCTs compared the efficacy of both cathodal and anodal tDCS with that of sham. In most of the cathodal stimulation trials, the target areas were the occipital regions, with reference on central or supraorbital areas. In anodal RCTs, the anode was usually placed above the motor cortical areas and the cathode on supraorbital areas. All RCTs adopted repeated sessions (from 5 to 28) at variable intervals, while the follow-up length spanned from 1 day up to 12 months. Efficacy results were variable but overall positive. According to the RoB-2 tool, only four of the 13 RCTs had a low risk of bias, while the others presented some concerns.
\end{abstract}

Conclusions: Both anodal and cathodal tDCS are promising for migraine prevention. However, there is a need for larger and rigorous RCTs and standardized procedures. Additionally, the potential benefits and targeted neurostimulation protocols should be assessed for specific subgroups of patients.

Keywords: Transcranial direct current stimulation, migraine, non-pharmacological treatment, migraine prevention

\footnotetext{
*Correspondence: simona.sacco@univaq.it

Neuroscience Section, Department of Applied Clinical and Biotechnological Sciences, University of L'Aquila, L'Aquila, Italy
} 


\section{Introduction}

Migraine preventive treatments are indicated in case of high-frequency, highly disabling episodes, or scarce response to acute treatments. Pharmacological treatments of different classes, including antihypertensives, antidepressants, anticonvulsants, onabotulinumtoxinA, and treatments acting on the calcitonin gene-related peptide, are suitable for most patients with migraine [1]. Neuromodulation techniques are an appealing complement or alternative to pharmacological treatment. Neuromodulation can be used in patients who prefer non-pharmacological management or who cannot be adequately managed with pharmacological treatment [2].

Migraine has a complex and still unclear pathophysiology involving several circuits of both the central and peripheral nervous system $[3,4]$. According to the most credited hypotheses, the activation of the trigeminovascular system, mostly pertaining to the peripheral nervous system, is the key event of migraine pain generation [5]; however, the central nervous system is also involved in the generation and perception of migraine [6]. The brain cortex of subjects with migraine is hyper-responsive to external stimuli [7], possibly due to altered functional connectivity with subcortical structures, including the thalamus [3]. The resulting "thalamo-cortical dysrhythmia" can be controlled by noninvasive neuromodulation techniques, including transcranial magnetic stimulation (TMS) and transcranial direct current stimulation (tDCS) [8]. tDCS is based on delivering a low-intensity current through the scalp by means of electrodes to modulate the state of polarization of the cerebral cortex; depending on the polarity of the electrical stimulation, tDCS can be either anodal or cathodal, whereas cathodal stimulation has a hyperpolarizing (inhibitory) effect and anodal stimulation has a depolarizing (excitatory) effect [9].

Several small randomized controlled trials (RCTs) showed the efficacy of different protocols of tDCS over sham stimulation for the prevention of migraine, as pointed out by previous systematic reviews and metaanalyses [10-13]. However, more and more high-quality RCTs of $\mathrm{tDCS}$ are being issued in recent years leading to a rapid evolution of the field. In those RCTs, the stimulation protocols widely differ, in the absence of any agreement. The International Headache Society published guidelines to improve and standardize the conduction of RCTs of neuromodulation for migraine prevention [14]; comparing those guidelines with the RCT methodology might help evaluating research in the field.

In the present review, we aimed at summarizing the knowledge we have so far on tDCS protocols for migraine prevention and providing insights and suggestions for the design of future trials.

\section{Methods}

We conducted a systematic review according to the 'Cochrane Handbook for Systematic Reviews of Interventions', Version 5.1.0 [15]; to report results, we followed the 'Preferred Reporting Items for Systematics Reviews and Meta-Analyses' (PRISMA) guidelines which were updated in 2020 [16]. The review was submitted to PROSPERO on September $24^{\text {th }}, 2021$.

\section{Search strategy and study selection}

We combined the keywords 'migraine', 'headache', 'transcranial direct current stimulation', and 'tDCS' and searched Pubmed, Scopus, and Web of Science on June 22nd, 2021. No additional filters were considered with the purpose of ensure high sensitivity of the search strategy. Retrieved references were managed with EndNote Free Web to remove duplicate publications.

Four raters (RO, VC, SR, GDA) selected the retrieved records in a two-step process, by first examining titles and abstracts and, subsequently, full texts of eligible references. In both steps, each record was assessed by two raters blinded to each other's evaluation. Titles and abstracts were evaluated in full text if respecting the following criteria: a) to be primary studies (reviews, editorials, letters, case reports, and case series were excluded) with published data; b) to include migraine patients; c) to include a tDCS intervention. When the abstract was not available, the reference was considered eligible and evaluated in full text. To be included in the systematic review, full texts of eligible articles had to be RCTs of a tDCS intervention compared with sham intervention and to report migraine-related outcomes such as headache frequency or acute medications intake. In both steps of article selection, all records were evaluated by two raters. In case of disagreement, a discussion among all the raters was performed to reach an agreement.

\section{Data extraction and analysis}

Four researchers (RO, VC, SR, GDA) used an electronic spreadsheet of Microsoft Excel for Windows to independently extract first author, publication year, number of included subjects, age and sex distribution, tDCS parameters (electrode size and position, current intensity, session length, number of sessions), assessment timepoints, and outcomes (headache/migraine frequency and intensity, acute drug consumption, other efficacy outcomes, adverse events). For each record, data were extracted by two raters blinded to each other's assessment.

Due to the heterogeneity in tDCS montages and schedules, outcome definitions, and assessment timepoints, no formal meta-analysis was performed. The quality of the included RCTs was assessed by assessing 
their adherence to the guidelines of the International Headache Society guidelines [14]. The risk of bias of each RCT was assessed according to the RoB-2 tool proposed by the Cochrane Collaboration [17]. This assessment was performed on the outcomes of migraine frequency, severity, and acute treatment utilization.

\section{Results}

\section{Literature search}

The database search identified 618 articles. After duplicate removal, 446 articles were screened for eligibility and 13 RCTs published from 2011 to 2021 were included in the review [18-30]. Details about study selection are reported in Fig. 1.

\section{Study populations}

Details of the patient populations included in the 13 RCTs and of their tDCS parameters are reported in Table 1 . The RCTs included 13 to 135 subjects; six trials included subjects with chronic migraine with or without medication overuse [19, 22-26], three included subjects with episodic migraine $[18,21,27]$, and one only women with menstrual migraine [30], while the remaining three RCTs included subjects with any migraine [20, 28, 29].

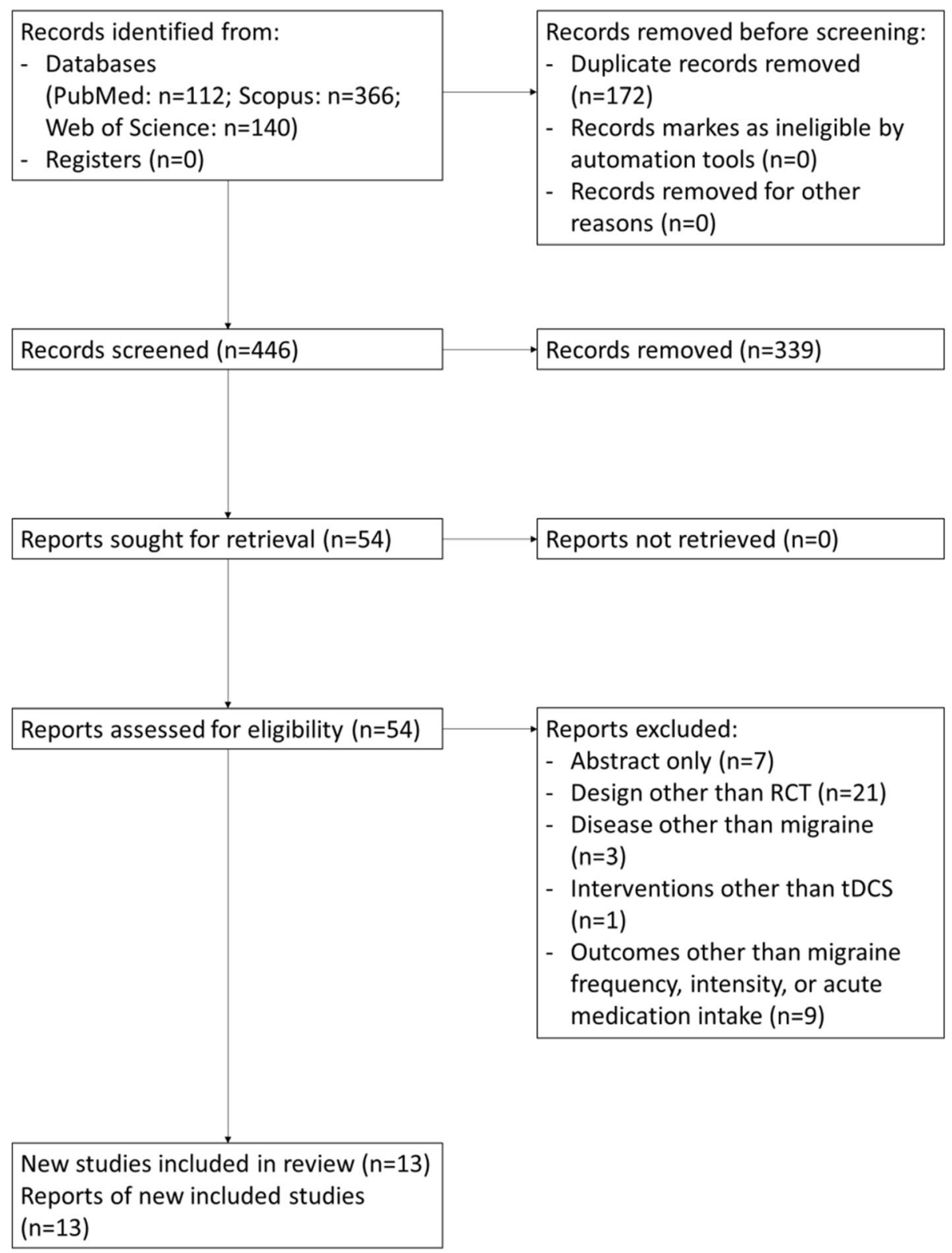

Fig. 1 PRISMA 2020 flowchart of study selection 


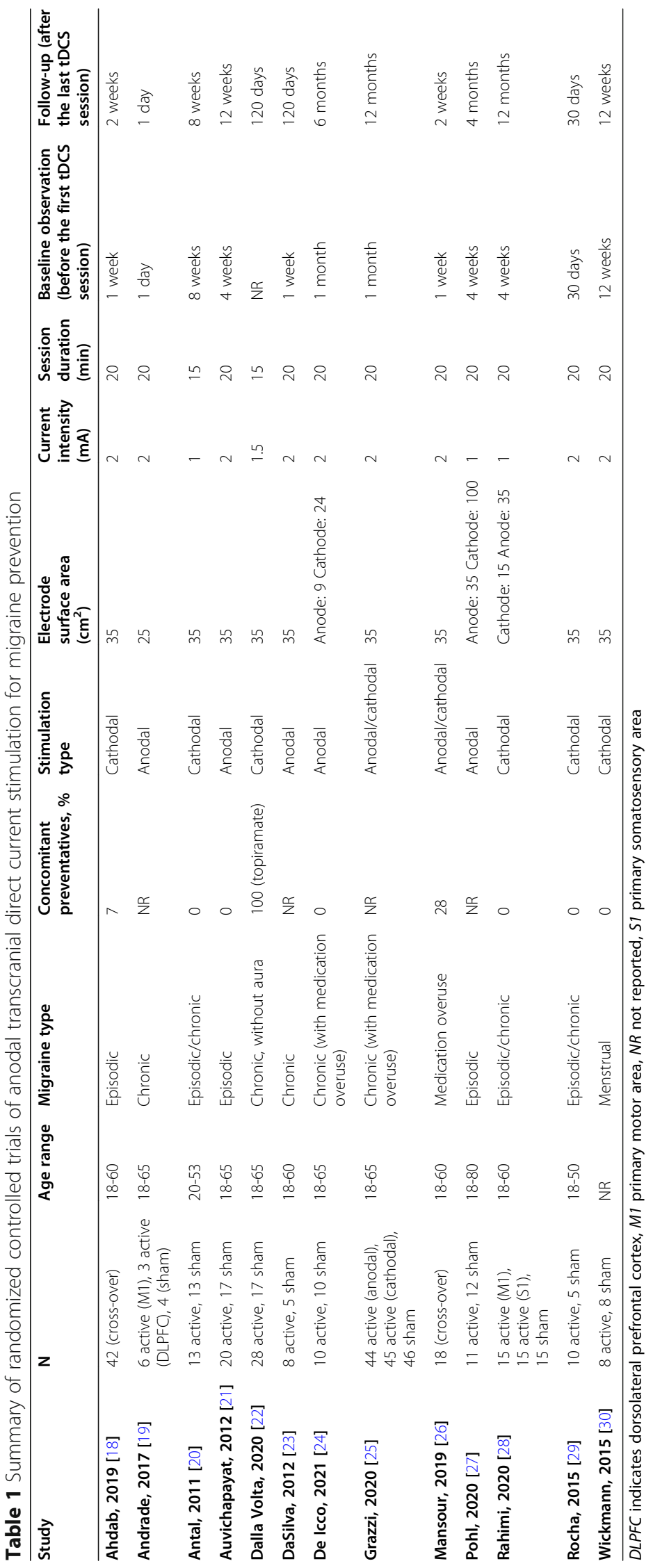


Only two RCTs reported stratified results by the presence of aura $[20,27]$.

\section{tDCS technical parameters, schedules, and assessment timepoints}

In most RCTs, the electrode surface area was $35 \mathrm{~cm}^{2}$, current intensity was $2 \mathrm{~mA}$, and the duration of each tDCS session was 20 minutes (Table 1). The number of sessions ranged from 5 to 28 , at variable distance between each other, while the length of follow-up ranged from 1 day to 12 months after the last tDCS session. Figure 2 reports details about the tDCS stimulation schedules.

\section{tDCS montages}

Six RCTs assessed the efficacy of cathodal tDCS [18, 20, $22,28-30]$, five assessed the efficacy of anodal tDCS [19, $21,23,24,27]$, and two of both techniques [25, 26]. Figure 3 reports the montages used for tDCS in each RCT.

\section{Cathodal tDCS}

In three RCTs, the cathode was positioned occipitally (Oz electrode according to the 10/20 international EEG system), with central reference ( $\mathrm{Cz}$ electrode) [20, 29, $30]$; in two trials, the cathode was positioned occipitally ipsilateral to the dominant side of migraine (O1 or $\mathrm{O} 2$ electrode), with contralateral supraorbital reference ( $\mathrm{Fp} 2$ or $\mathrm{Fp} 1$ electrodes) $[18,26]$; in one trial, the cathode was placed on the right M1 (C4 electrode), with left supraorbital reference (Fp1 electrode) [25]; in a further trial, the cathode was positioned above the right M1 (C4 electrode) or primary sensory area (S1; CP4 electrode in the 10/10 EEG system) with extracranial reference [28]; in the remaining RCT, the position of the cathode was variable and determined by thermography, with the identification of a "cold patch" over the scalp, with extracranial reference [22].

\section{Anodal tDCS}

In four RCTs of anodal tDCS, the anode was positioned above $\mathrm{M} 1$, with varying sides (C3 or $\mathrm{C} 4$ electrodes), with contralateral supraorbital reference (Fp2 or $\mathrm{Fp} 1$ electrodes) [21, 23, 24, 31]; one RCT placed anodes either above the left M1 or above the left dorsolateral prefrontal cortex (DLPFC; C3 or F3 electrodes, respectively), with contralateral supraorbital reference (Fp2) [19]; one RCT placed the anode over the left DLPFC (F3 electrode), with reference on the opposite side (F4 electrode) [26]; a further trial positioned the anode on the right M1 (C4 electrode) with left supraorbital reference (Fp1 electrode) [25];

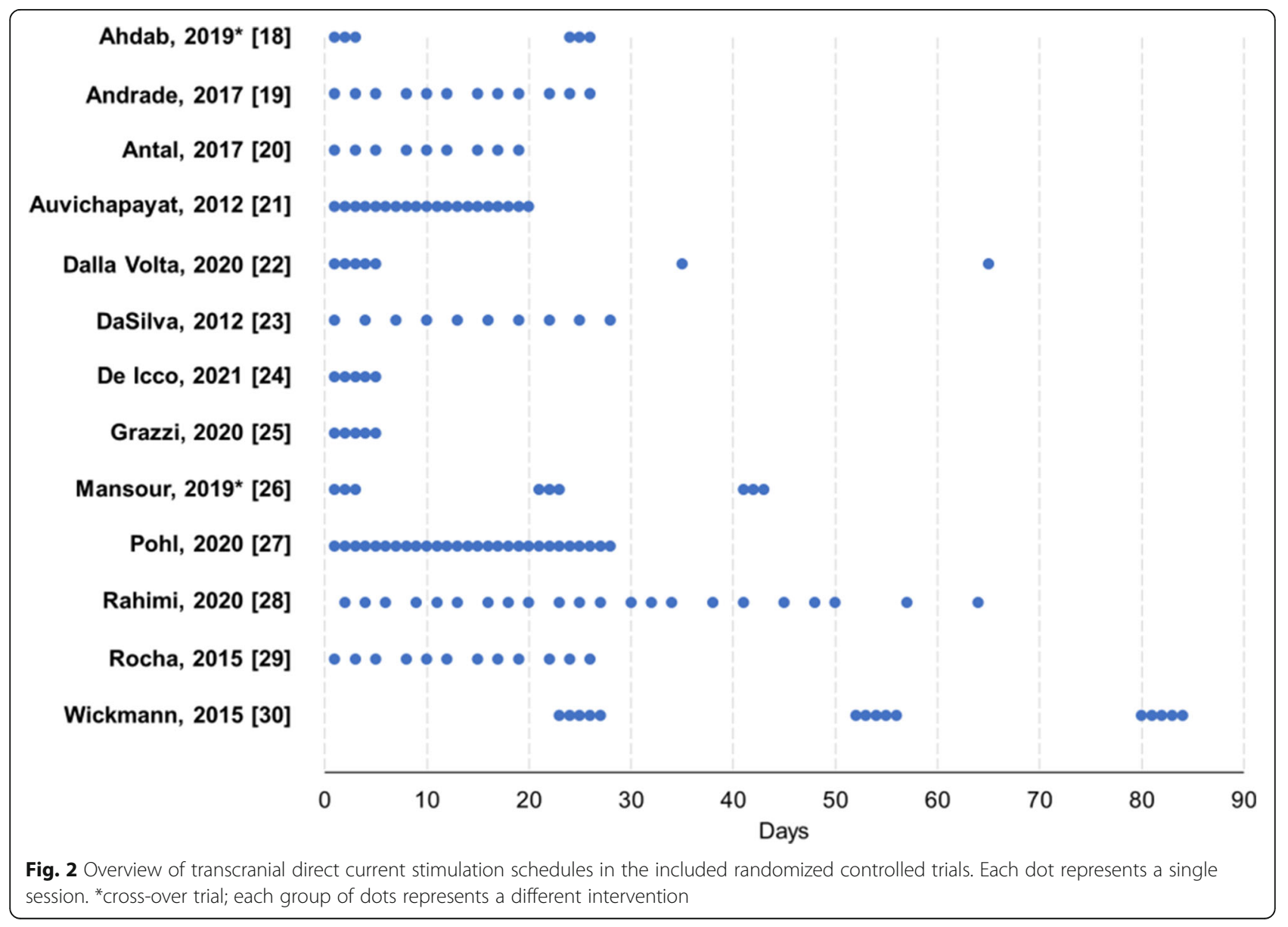




\section{Cathodal}

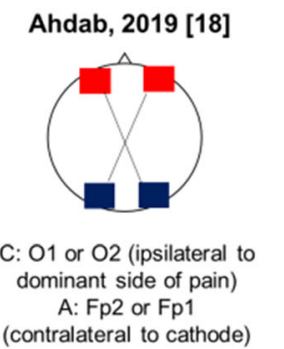

Mansour, 2019* [26]

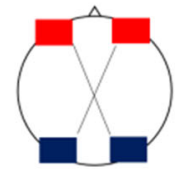

C: $\mathrm{O} 1$ or $\mathrm{O} 2$ (ipsilateral to dominant side of pain) A: Fp2 or Fp1 (contralateral to cathode)
Antal, 2017 [20]

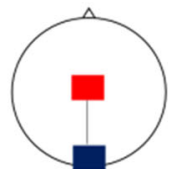

C: $\mathrm{Oz}$ $\mathrm{A}: \mathrm{Cz}$
Dalla Volta, 2020 [22]

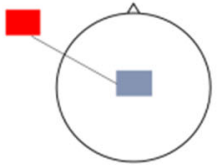

C: Cold patch of scalp identified through thermography (variable position) A: extracranial
Grazzi, 2020* [25]

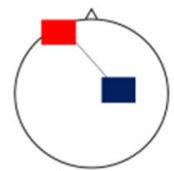

C: C4
Rahimi, 2020 [28]

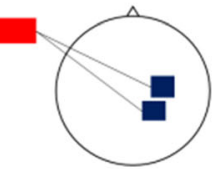

C: $\mathrm{C} 4$ or a point between $\mathrm{C} 4$ and $\mathrm{CP}_{4}{ }^{* *}$ A: upper part of left arm

\section{Rocha, 2015 [29]}

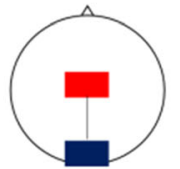

C: $\mathrm{Oz}$

$\mathrm{A}: \mathrm{Cz}$
Wickmann, 2015 [30]

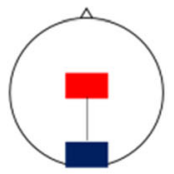

C: $\mathrm{Oz}$

\section{Anodal}

\section{Andrade, 2017 [19]}

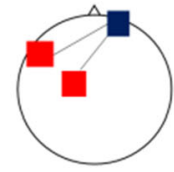

A: $\mathrm{F} 3$ or $\mathrm{C} 3$

C: Fp2
Auvichapayat, 2012 [21]

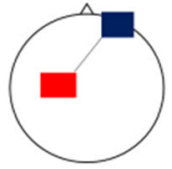

A: $\mathrm{C} 3$

C: $\mathrm{Fp} 2$
DaSilva, 2012 [23]

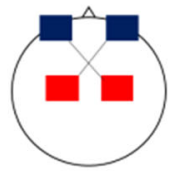

A: C3 or C4 (contralateral to dominant side of pain) $\mathrm{C}$ : Fp2 or Fp1 (contralateral to anode)
De Icco, 2021 [24]

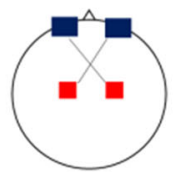

A: C3 or C4 (contralateral to dominant side of pain) C: Fp2 or Fp1 (contralateral to anode)
Grazzi, 2020* [25]

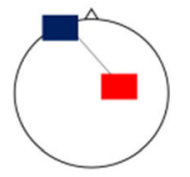

A: C4
Mansour, 2019* [26]

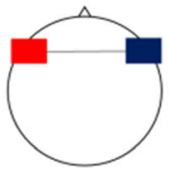

A: F3
Pohl, 2020 [27]

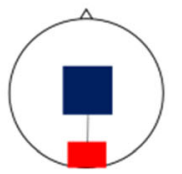

A: $\mathrm{Oz}$

Fig. 3 Overview of transcranial direct current stimulation montages in the included randomized controlled trials. Blue squares/rectangles indicate cathodes, while red squares/rectangles indicate anodes. Electrodes' shape and dimension reflect the differences in RCTs stimulation setups. Solid lines connect the electrodes pairs that have been tested in different stimulation conditions in each RCT. The positions of anodes and cathodes are identified according to the international 10/20 electroencephalographic system. *This study assessed both cathodal and anodal stimulation. **10/10 electroencephalographic system

the remaining $\mathrm{RCT}$ placed the anode above the occipital areas $(\mathrm{Oz}$ electrode) with central reference $(\mathrm{Cz}$ electrode) [27].

\section{Adherence to guideline standards}

Figure 4 displays the characteristics of the included RCTs compared with those required by international guidelines
[14]. All RCTs provided an adequate definition of migraine, had an adequate trial design, and reliably monitored patients' outcomes by using headache diaries. Of note, none of the RCTs was multicenter. Five RCTs did not report details about the concomitant preventive treatments for migraine taken by patients $[18,19,23,25,27]$, while only two RCTs performed an assessment of blinding [19, 24]. 


\begin{tabular}{|c|c|c|c|c|c|c|c|c|c|c|c|c|c|}
\hline Criterion & $\begin{array}{c}\text { Ahdab } \\
2019[18]\end{array}$ & $\begin{array}{l}\text { Andrade, } \\
2017[19]\end{array}$ & $\begin{array}{l}\text { Antal, } \\
2011 \text { [20] }\end{array}$ & $\begin{array}{l}\text { Auvichapayat, } \\
2012[21]\end{array}$ & $\begin{array}{l}\text { Dallla Volta, } \\
\text { 2020 [22] }\end{array}$ & 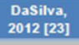 & $\begin{array}{l}\text { De loco, } \\
2021[24]\end{array}$ & $\begin{array}{c}G[a z 23, \\
2020[25]\end{array}$ & $\begin{array}{l}\text { Mansour, } \\
2019[26]\end{array}$ & $\begin{array}{l}\text { Ponh, } \\
2020[27]\end{array}$ & $\begin{array}{l}\text { Rahimi, } \\
2020[28]\end{array}$ & $\begin{array}{l}\text { Rocha, } \\
2015[23]\end{array}$ & 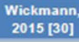 \\
\hline \multicolumn{14}{|l|}{ Subject Selection } \\
\hline Migraine definition & 은 & 0 & 0 & O & 은 & 은 & 0 & 0 & 0 & 0 & 0 & 0 & 0 \\
\hline Age at onset & 0 & 0 & 0 & 0 & 0 & 0 & 0 & 0 & 0 & 0 & 0 & 0 & 0 \\
\hline Age at entry & 0 & 0 & 0 & 0 & 0 & 0 & 0 & 0 & 0 & - & 0 & 0 & 0 \\
\hline Duration of migraine & 0 & 0 & 0 & 0 & - & 은 & 0 & 0 & 0 & 0 & 0 & 0 & - \\
\hline Sex & 0 & 0 & 0 & 0 & 0 & 0 & 0 & 0 & 0 & o & 0 & 0 & - \\
\hline Concomitant drug use & 0 & 0 & 0 & 0 & 0 & 0 & 0 & 0 & 0 & 0 & 0 & 0 & 0 \\
\hline \multicolumn{14}{|l|}{ Study design } \\
\hline Study center & 0 & 0 & 0 & 0 & 0 & 0 & 0 & 0 & 0 & 0 & 0 & 0 & - \\
\hline Training & 0 & 0 & 0 & 0 & 0 & 0 & 0 & 0 & 0 & 0 & 0 & 0 & 0 \\
\hline Blinding & 0 & 0 & 0 & 0 & 0 & 0 & 0 & 0 & 0 & - & 0 & 0 & 0 \\
\hline Controls & 0 & 0 & 인 & 0 & 0 & 은 & - & 0 & 0 & - & 0 & 0 & 0 \\
\hline Design types & 0 & 0 & 0 & 0 & 0 & 0 & 0 & 0 & 0 & - & 0 & 0 & 0 \\
\hline Randomization & 0 & 0 & - & 0 & 0 & 0 & 0 & 0 & 0 & O & ○ & 0 & 0 \\
\hline Stratification & - & 0 & 0 & - & - & - & - & - & - & 0 & - & - & - \\
\hline $\begin{array}{l}\text { Intention-to-Treat / / modified } \\
\text { Intention-to-Ireata }\end{array}$ & - & ○ & - & 0 & 0 & 0 & 0 & 0 & - & 0 & 0 & 0 & 0 \\
\hline Duration of the study & - & - & - & 0 & - & 0 & 0 & 0 & - & 0 & 0 & - & 0 \\
\hline \multicolumn{14}{|l|}{ Result Evaluation } \\
\hline Statistics & 0 & 0 & 0 & 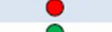 & 0 & 0 & 0 & 0 & 은 & 은 & 은 & 은 & 은 \\
\hline Monitoring & 0 & 0 & 0 & 0 & 0 & 0 & 0 & 0 & 0 & 은 & 0 & 0 & 0 \\
\hline Adverse events & 0 & 0 & 0 & 0 & 0 & - & 0 & 0 & - & 0 & - & - & 0 \\
\hline Blinding assessment & - & 0 & 0 & - & - & - & 0 & - & - & - & - & - & 0 \\
\hline \multicolumn{14}{|l|}{ Other } \\
\hline $\begin{array}{l}\text { Participation in multiple } \\
\text { trials }\end{array}$ & 0 & 0 & 0 & 0 & 0 & 0 & 0 & 0 & 0 & 0 & 0 & 0 & 0 \\
\hline
\end{tabular}

Fig. 4 Adherence of available randomized controlled trials on transcranial direct current stimulation to the guideline recommendations for clinical trials of neuromodulation devices for the treatment of migraine in adults [14]. A green circle indicates that the criterion was met; a red circle, that it was not met; an amber circle, that it was not specified

The outcomes reported by the included RCTs mostly referred to migraine or headache days, headache intensity, and acute treatment utilization; questionnaires to assess patient-reported outcomes, such as disability and quality of life, were only used by few RCTs; no RCT assessed the usability of tDCS devices or quantified their costs (Figure 4).

\section{Efficacy and safety}

A schematic representation of the efficacy data of included RCTs is shown in Figure 5, while detailed efficacy data are reported in Supplementary file S1. Most RCTs showed a significantly greater decrease in headache frequency, intensity, and acute medication consumption in the active groups compared with sham. Results did not

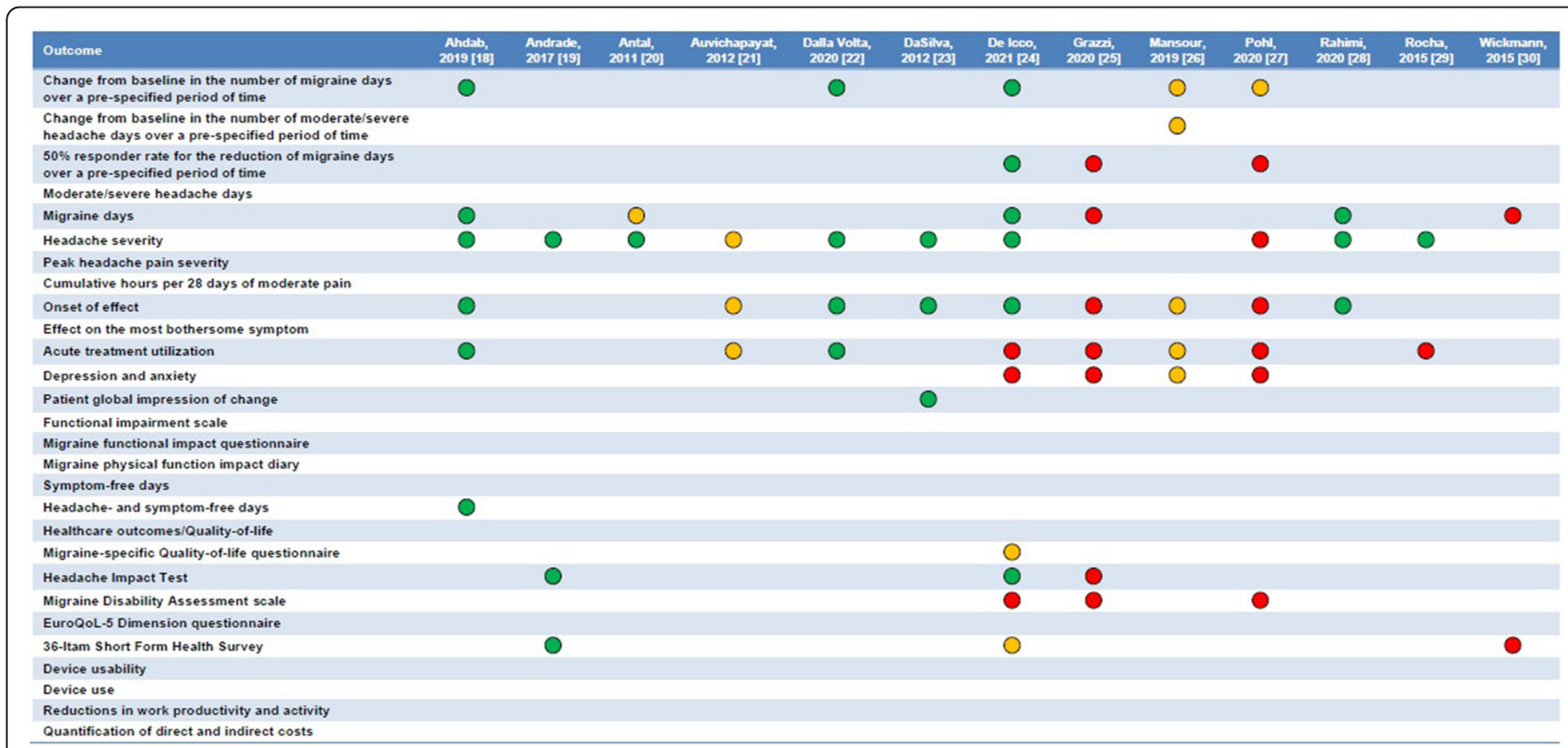

Fig. 5 Results of the available randomized controlled trials comparing transcranial direct current stimulation to sham procedure. The list is taken from the guidelines of clinical trials of neuromodulation devices for the treatment of migraine in adults [14]. More detailed quantitative reports are provided in Supplemental file 1. Green circles indicate that tDCS was entirely superior to sham, amber circles that tDCS was partially superior to sham, red circles that tDCS was note superior to sham; cells were left empty if outcomes were not reported 
show any difference between active tDCS and sham in the RCT of anodal stimulation over the occipital areas [27], in which only headache intensity decreased in the active group compared with sham, in the trial on patients with medication overuse [24], and in the RCT of tDCS in menstrual migraine [30]. The presence of aura did not influence outcomes in one RCT [27], while in another RCT patients with migraine with aura had a higher advantage than those without aura in terms of headache intensity reduction [20]. In one RCT the effect of tDCS waned after 12 weeks [21], while in another RCT the effect was detectable up to 6 months after a 5-day intervention [24]. In all RCTs, adverse events mostly occurred during the tDCS procedure and were mild and transient. Figure 6 reports the difference between active and sham groups in the four most reported adverse events, i.e., tingling, itching, headache, and pain; more details are provided in Supplementary file S2. Notably, none of the RCTs found differences in adverse events between active and sham groups. No serious adverse event was reported by any RCT.

\section{Risk of bias}

Details of the risk of bias assessment are reported in Supplementary file S3. Only four of the 13 RCTs included had a low risk of bias $[19,23-25]$, while the remaining nine RCTs presented some concerns [18, 20-22, 26-30]; no study had a high risk of bias.

\section{Discussion}

Our systematic review showed that either cathodal or anodal tDCS can have some benefit for the prevention of migraine; however, the available RCTs are mostly pilot studies not fulfilling all the quality criteria. Overall, the technique proved to be beneficial for the decrease in migraine frequency, intensity, and acute drug utilization; however, the tDCS schedules and montages used in the RCTs were heterogeneous. Hence, it is not possible to date to recommend any tDCS approach that best suits patients with migraine.

It is important to promote consensus over tDCS protocols to spread the use of this technique for clinical purposes in patients with migraine. For this reason, the primary aim of this study was to provide a systematic review focused on methodological criteria of the available studies, and particularly on stimulation protocols and patient selection. Across the available RCTs, many factors of heterogeneity should be considered, referring to patient population, stimulation protocols, assessment timepoints, and outcomes.

\section{Patients' characteristics}

The available RCTs included either subjects with episodic $[18,21,27]$ or with chronic migraine [19, 22, 23], while some trials selected subjects with medication overuse [24-26]; three additional RCTs included subjects

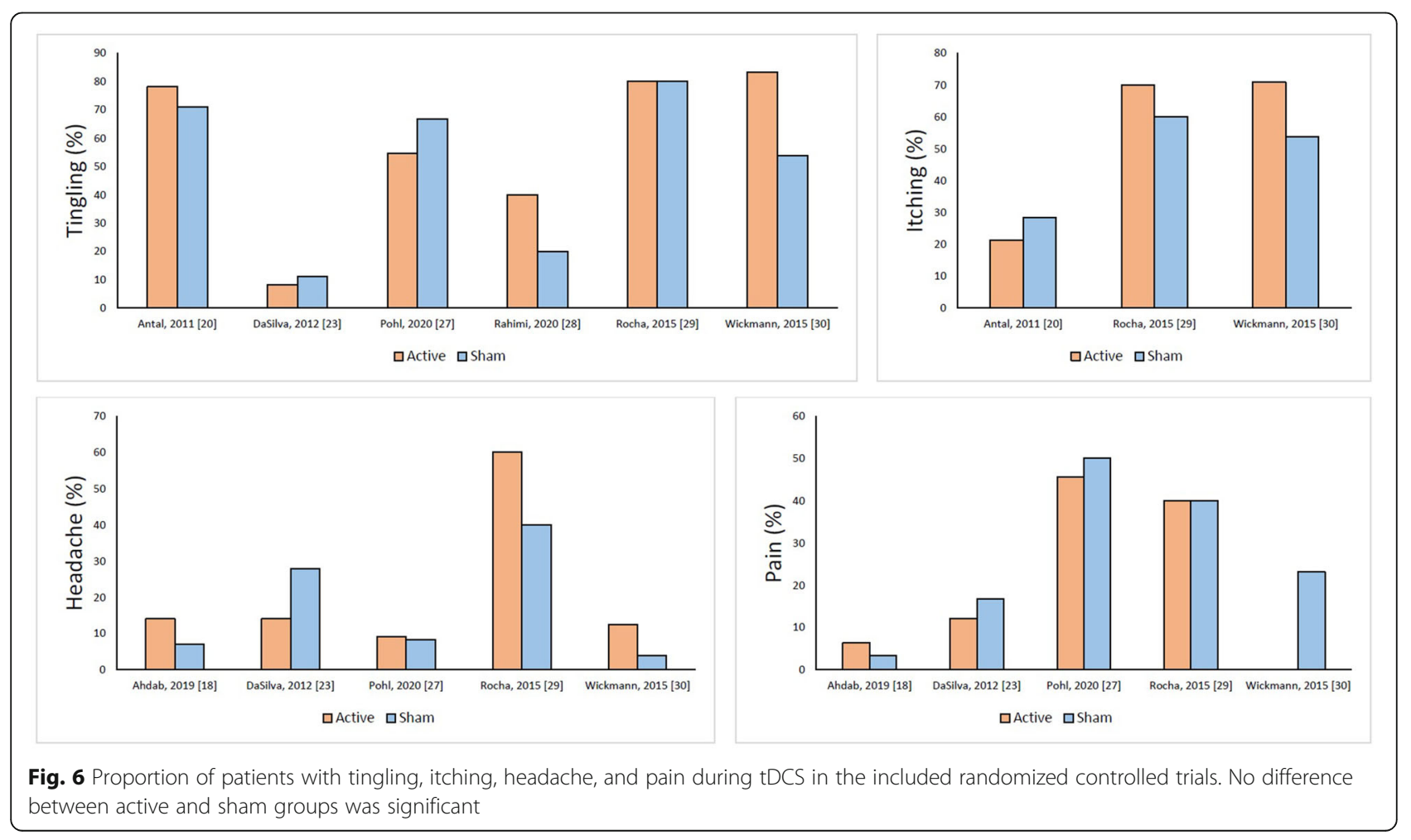


with both episodic and chronic migraine [20, 28-30]. In all those populations, tDCS led to some therapeutic effect. However, the RCTs were overall underpowered to allow any subgroup analysis. Only two RCTs performed exploratory subgroup analyses in patients with and without aura, showing similar efficacy in the two subgroups [20, 27].

The available RCTs either excluded patients with concomitant migraine preventive medication [20, 21, 24, 26, 28-30] or did not report the proportion of patients treated with preventive drugs $[19,23,25,27]$; only in one RCT tDCS was used in combination with topiramate in all patients [22]. Even in the RCT on patients using topiramate, tDCS showed some efficacy compared with sham. Besides, in two RCTs tDCS enhanced the effect of detoxication for patients with medication overuse $[24,26]$. According to guideline recommendations, the inclusion in neuromodulation RCTs of patients with concomitant preventive medication should be liberal, provided that patients do not change their medication for at least 3 months [14]. In the absence of better data, tDCS can be considered suitable for patients with migraine irrespective of their concomitant treatments, even if most of the evidence is available for patients without concomitant treatments.

A further point to consider for patient selection are prior preventive treatment failures. Considering those failures has become increasingly important in recent years due to the eligibility criteria for treatments acting on the calcitonin gene-related peptide [32, 33]. Most RCTs did not report the proportion of patients who had failed preventive treatments for their migraine; one RCT only included patients who had not tried any preventive treatment [21], while one further RCT only included patients with three or more failures [24]. Our review shows that there are currently no available data to conclude whether the efficacy of tDCS changes according to previous preventive treatment failures. In our opinion, tDCS can be best offered to patients with frequent and severe migraine who are resistant or refractory to preventive medication [2]. However, the technique might also be an option for patients who refuse drugs or with contraindications to migraine preventive treatments.

\section{Stimulation schedules and assessment timepoints}

The RCTs included in the present systematic review adopted different stimulation schedules (Figure 2); there is no direct comparison between different protocols, leaving uncertainty about the optimal stimulation protocol over time. In clinical practice, the number of possible consecutive tDCS sessions is limited by the fact that tDCS is performed by trained personnel during working hours. Self-administered tDCS would obviate to this problem. However, the results of the only RCT assessing the efficacy of self-administered tDCS [27] were worse than those of other RCTs (Figure 5), suggesting that self-administered tDCS should still be tested.

A question that may arise in clinical practice is about the duration of tDCS effect, in order to plan repetitive stimulation. The duration of effect of tDCS was noted up to 12 months in one RCT [25]; however, some other trials showed a waning effect of tDCS over 3-4 months $[18,21]$. Notably, the RCT with the worst efficacy results was also the one with the longest follow-up [25]. The duration of tDCS effect might be influenced by the stimulation schedule. However, it is difficult to estimate the number of consecutive sessions that ensure the longest duration of tDCS effect, as each of the available RCTs adopted a different approach.

\section{Trial methodology}

Overall, the quality of RCTs of tDCS for migraine prevention is not high. Only few of the outcomes recommended by international guidelines [14] were reported by RCTs (Figure 4). RCTs mostly focused on headache intensity and frequency; outcomes that are often reported by pharmacological RCTs, such as the proportion of patients with a $\geq 50 \%$ decrease in migraine days compared with baseline, were seldom reported (Figure 4). International guidelines recommend reporting patientreported outcomes, together with outcomes related to device usability and costs [14], which were not reported by the RCTs of tDCS. There is a need for a more thorough assessment of migraine-related outcomes in neuromodulation trials, to better compare their efficacy with that of drugs and integrate those non-pharmacological treatments into clinical practice guidelines.

Most of the included RCTs had some methodological concerns according to the Cochrane RoB-2 tool; only four RCT had a low risk of bias (Supplemental file S1). Some concerns in the risk of bias were detected in most assessment domains. Several RCTs were conceived as pilot trials, while there is a need for more complete RCTs compliant with the most recent guidelines.

\section{Stimulation sites and neuroanatomical targets}

To date, the preferable target of tDCS for migraine prevention still has to be determined. Cathodal, i.e., inhibitory, tDCS mostly targeted the occipital cortex (Figure 3). The visual cortex is hyper-responsive in patients with migraine compared with non-migraineurs, as shown by experimental evidence $[34,35]$. Therefore, the rationale of hyperpolarizing the visual cortex of patients with migraine by cathodal electrical stimulation is to avoid the hyper-responsivity of the visual cortex. On the other hand, RCTs performing anodal (excitatory) tDCS targeted the M1 or DLPFC (Figure 3) with the aim of inducing a reflex inhibition of subcortical pain-generating 
areas, centered on the thalamus; this approach has been already adopted in RCTs of tDCS for fibromyalgia and other chronic pain conditions [36]. More specifically, the stimulation of the M1 causes a direct inhibition of the thalamus [37], while excitatory stimulation of the DLPFC can inhibit the midbrainmedial thalamic pathway [38].

The available literature cannot suggest any tDCS montage that is better than others at preventing migraine. Each montage targets specific neural structures, all potentially implied in migraine pathogenesis [36]. Preliminary identification of the neuroanatomical targets most suitable to each patient would be an important advance for a wider employment and better results of tDCS.

\section{The role of functional tests}

In our opinion, a first step toward the identification of adequate targets for tDCS is studying the effect of this intervention with functional tests; however, those tests were rarely used by the available RCTs and highly heterogenous in nature. Referring to the three RCTs included in the present review, one verified the neurophysiologic effect of tDCS by using EEG [24], the second with the detection of phosphene threshold [29], and the third with a computational model based upon functional neuroimaging [23]. At EEG, anodal tDCS of the primary motor cortex resulted in increased alpha power over the occipital cortex; this effect could be attributed to either direct modulation of cortical activity or to the decrease of the thalamo-cortical dysrhythmia observed in migraineurs [24]. Compared with EEG, results related to phosphene threshold were more controversial. Phosphene threshold is lower in migraineurs compared with nonmigraineurs due to higher responsiveness of visual cortex [39]. The RCT included in the present review confirmed that phosphene threshold, assessed through transcranial magnetic stimulation, was lower in migraineurs than in non-migraineurs; however, occipital cathodal tDCS was not able to increase that threshold, suggesting that cortical hyper-responsiveness persisted in migraineurs [29]. In another RCT, a computational model showed that anodal tDCS over the motor cortex was able to generate electrical fields not only in the cortex, but also in the insula, cingulate cortex, thalamus, and brainstem, all of which contain circuits implied in the genesis of migraine [23].

Functional tests could be useful not only to monitor the effects of tDCS, but also to select patients who might respond to the treatment. In one RCT a neurophysiologic test, i.e., low phosphene threshold of the visual cortex, was adopted as selection criteria for cathodal tDCS [29]. An open-label study [40] that was not included in the present review due to its design also showed the effectiveness of cathodal tDCS over the visual cortex in selected patients with abnormal excitability of the visual cortex. A further RCT selected a "cold patch" of the head by thermography as the target of cathodal tDCS [22], as previous evidence showed that those cold patches could be related to migraine generation; however, there was no replication of this technique in further RCTs. Functional tests could help improving not only the efficacy of neuromodulation interventions, but also our understanding of the pathophysiology of migraine.

\section{Is the presence of aura a selection criterion for tDCS?}

A further element that could determine the response of migraine to neuromodulation is the presence of aura. Aura is assumed to be the clinical correlate of cortical spreading depression (CSD), a spreading wave of depolarization that is followed by a decrease in neuronal activity and blood flow [41-43]. There is indirect experimental evidence that CSD is linked to aura in patients with migraine [44, 45]. Therefore, the activation of brain cortex could be a prominent phenomenon in migraine with aura, while in migraine without aura subcortical structures, including the thalamus, hypothalamus, and brainstem can play a prominent role $[4,46]$. Evidence suggests that neuromodulation techniques such as transcranial magnetic stimulation might act better on migraine with aura than on migraine without aura [47, 48]. Surprisingly, tDCS had similar effect sizes in both migraine with and without aura in two RCTs [20, 27]. This lack of difference might depend on the low number of patients included in RCTs, too low to allow the detection of significant effects. As a further point, patients might have low-frequency auras, therefore hindering a specific effect of tDCS on aura.

\section{Suggestions for future research}

According to the results of the available trials, it is not possible to determine which approach is the best to prevent migraine. Nevertheless, the positive results of most RCTs encourage new studies to provide conclusive evidence. The available RCTs present some issues that could be solved by further studies; the unmet needs of tDCS research in the field of migraine are summarized in Figure 7. Referring to methods, there is a need for multicenter RCTs with adequate sample size calculations, strategies to control for confounding factors and incomplete data, and to perform subgroup analyses for the different populations of interest. The range of reported outcomes should also be expanded to include patient-reported outcomes and cost-effectiveness assessments, as suggested by international guidelines [14]. Referring to tDCS schedules, RCTs suggested a precise range of current intensity ( 1 to $2 \mathrm{~mA}$ ) and duration of each session (15-20 minutes); however, there is no 


\title{
Trial protocols
}

- Multicenter trials

- Further outcomes (patient-reported, cost-effectiveness)

- Adequate sample size/power

- Long-term follow-up

- Correction for confounders

\section{Populations}

- Subgroup analyses according to migraine characteristics (aura, medication overuse, resistance to preventive medication)

- Role of comorbidities

\author{
Technical issues \\ - Comparison between different schedules \\ - Comparison between different montages
}

\section{Neurophysiology tests \\ - Patient selection \\ - Outcome assessment}

Fig. 7 Unmet needs in transcranial direct current stimulation trials for migraine prevention

consensus over the optimal number of sessions and time interval between them. Future RCTs should compare different schedules and monitor the onset of tDCS effect and its duration over time. Referring to tDCS montages, excitatory stimulation is best centered over motor areas, while inhibitory stimulation is best centered over the visual cortex. Because both the cathode and anode of tDCS can perform a stimulation, positioning the anode (excitatory stimulation) over the motor cortex and the cathode (inhibitory stimulation) over the visual cortex might stimulate at once most of the brain areas that are relevant for migraine prevention. The help of neurophysiological tests to select appropriate patient groups and montages, as well as to monitor tDCS outcomes, could largely improve research in the field and consequently clinical practice.

\section{Strengths and limitations}

The present review systematically reported the results of the available RCTs on tDCS for migraine prevention and is a substantial update to previous reviews [10-13], as several important RCTs were published in the last two years. We chose to maintain a conservative approach by not performing a meta-analysis, given the remarkable heterogeneity across RCTs. Despite those strengths, the inclusion of RCTs only could constitute a limitation, as observational studies could have added important information to the topic. Besides, the heterogeneity of RCTs did not allow to draw definite conclusions on the best possible tDCS protocol for the prevention of migraine. 


\section{Conclusions}

tDCS is a non-invasive neuromodulation technique highly promising to effectively prevent migraine without relevant harms to patients. An advantage of tDCS is its non-pharmacological nature, which makes it suitable for patients with comorbidities or poor tolerance to pharmacological treatments. Besides, tDCS acts on the central nervous system thus potentially being synergic with migraine preventive drugs that mostly act at a peripheral level. However, there is still an unmet need of high-quality RCTs supporting a wider use of tDCS and a need for standardization of the procedure.

\section{Supplementary Information}

The online version contains supplementary material available at https://doi. org/10.1186/s10194-021-01361-0.

Additional file 1. Summary of results of included trials.

Additional file 2. Adverse events in the included trials.

Additional file 3. Risk of bias analysis.

\section{Acknowledgements}

None.

\section{Authors' contributions}

$\mathrm{RO}, \mathrm{SS}$, and ADA conceived the study. RO, VC, SR, GDA performed literature search and data interpretation. RO drafted the work. All authors substantively reviewed the manuscript and approved the submitted version.

\section{Funding}

This work was supported by a grant to ADA and RO from the University of L'Aquila (Prot. n. 80798).

\section{Availability of data and materials}

Data sharing is not applicable to this article as no datasets were generated or analysed during the current study.

\section{Declarations}

Ethics approval and consent to participate Not applicable.

\section{Consent for publication}

Not applicable.

\section{Competing interests}

RO reports personal fees from Novartis, Teva, and Eli Lilly, and had nonfinancial relationships with Allergan/AbbVie, Novartis and Teva. SS reports personal fees and nonfinancial support from Allergan, Abbott, Eli Lilly, Lundbeck, Novartis, and Teva; personal fees from Medscape; and other from Bayer, Pfizer, Medtronic, Starmed, Bristol-Myers Squibb, and Daiichi Sankyo. The remaining Authors declare no competing interests.

Received: 11 October 2021 Accepted: 17 November 2021

Published online: 27 November 2021

\section{References}

1. Burch R (2021) Preventive Migraine Treatment. Continuum (Minneap Minn) 27(3):613-632

2. Sacco S, Braschinsky M, Ducros A, Lampl C, Little P, van den Brink AM et al (2020) European headache federation consensus on the definition of resistant and refractory migraine : Developed with the endorsement of the European Migraine \& Headache Alliance (EMHA). J Headache Pain 21(1):76

3. Charles A (2018) The pathophysiology of migraine: implications for clinical management. Lancet Neurol 17(2):174-182
4. Goadsby PJ, Holland PR, Martins-Oliveira M, Hoffmann J, Schankin C, Akerman S (2017) Pathophysiology of Migraine: A Disorder of Sensory Processing. Physiol Rev 97(2):553-622

5. Ashina M, Hansen JM, Do TP, Melo-Carrillo A, Burstein R, Moskowitz MA (2019) Migraine and the trigeminovascular system-40 years and counting. Lancet Neurol 18(8):795-804

6. Ashina M (2020) Migraine. N Engl J Med 383(19):1866-1876

7. de Tommaso M, Ambrosini A, Brighina F, Coppola G, Perrotta A, Pierelli F et al (2014) Altered processing of sensory stimuli in patients with migraine. Nat Rev Neurol 10(3):144-155

8. Coppola G, Di Lorenzo C, Serrao M, Parisi V, Schoenen J, Pierelli F (2016) Pathophysiological targets for non-pharmacological treatment of migraine. Cephalalgia. 36(12):1103-1111

9. Lambru G, Lantéri-Minet M (2019) Neuromodulation in headache and facial pain management: principles, rationale and clinical data. Springer Nature

10. Shirahige L, Melo L, Nogueira F, Rocha S, Monte-Silva K (2016) Efficacy of Noninvasive Brain Stimulation on Pain Control in Migraine Patients: A Systematic Review and Meta-Analysis. Headache. 56(10):1565-1596

11. Stilling JM, Monchi O, Amoozegar F, Debert CT (2019) Transcranial Magnetic and Direct Current Stimulation (TMS/tDCS) for the Treatment of Headache: A Systematic Review. Headache. 59(3):339-357

12. Feng $Y$, Zhang B, Zhang J, Yin $Y$ (2019) Effects of Non-invasive Brain Stimulation on Headache Intensity and Frequency of Headache Attacks in Patients With Migraine: A Systematic Review and Meta-Analysis. Headache. 59(9):1436-1447

13. Moisset X, Pereira B, Ciampi de Andrade D, Fontaine D, Lanteri-Minet M Mawet J (2020) Neuromodulation techniques for acute and preventive migraine treatment: a systematic review and meta-analysis of randomized controlled trials. J Headache Pain 21(1):142

14. Tassorelli C, Diener HC, Silberstein SD, Dodick DW, Goadsby PJ, Jensen RH et al (2021) Guidelines of the International Headache Society for clinical trials with neuromodulation devices for the treatment of migraine. Cephalalgia::3331024211010413

15. Higgins J, Thomas JE (2021) Cochrane Handbook for Systematic Reviews of Interventions. The Cochrane Collaboration

16. Page MJ, McKenzie JE, Bossuyt PM, Boutron I, Hoffmann TC, Mulrow CD et al (2021) The PRISMA 2020 statement: An updated guideline for reporting systematic reviews. Int J Surg 88:105906

17. Sterne JAC, Savovic J, Page MJ, Elbers RG, Blencowe NS, Boutron I et al (2019) RoB 2: a revised tool for assessing risk of bias in randomised trials. BMJ. 366:14898

18. Ahdab R, Mansour AG, Khazen G, El-Khoury C, Sabbouh TM, Salem M et al (2019) Cathodal Transcranial Direct Current Stimulation of the Occipital cortex in Episodic Migraine: A Randomized Sham-Controlled Crossover Study. J Clin Med 9(1)

19. Andrade SM, de Brito Aranha REL, de Oliveira EA, de Mendonca C, Martins WKN, Alves NT et al (2017) Transcranial direct current stimulation over the primary motor vs prefrontal cortex in refractory chronic migraine: A pilot randomized controlled trial. J Neurol Sci 378:225-232

20. Antal A, Kriener N, Lang N, Boros K, Paulus W (2011) Cathodal transcranial direct current stimulation of the visual cortex in the prophylactic treatment of migraine. Cephalalgia. 31(7):820-828

21. Auvichayapat $P$, Janyacharoen $T$, Rotenberg A, Tiamkao S, Krisanaprakornkit T. Sinawat S et al (2012) Migraine prophylaxis by anodal transcranial direct current stimulation, a randomized, placebo-controlled trial. J Med Assoc Thail 95(8):1003-1012

22. Dalla Volta G, Marceglia S, Zavarise P, Antonaci F (2020) Cathodal tDCS Guided by Thermography as Adjunctive Therapy in Chronic Migraine Patients: A Sham-Controlled Pilot Study. Front Neurol 11:121

23. Dasilva AF, Mendonca ME, Zaghi S, Lopes M, Dossantos MF, Spierings EL et al (2012) tDCS-induced analgesia and electrical fields in pain-related neural networks in chronic migraine. Headache. 52(8):1283-1295

24. De Icco R, Putorti A, De Paoli I, Ferrara E, Cremascoli R, Terzaghi M et al (2021) Anodal transcranial direct current stimulation in chronic migraine and medication overuse headache: A pilot double-blind randomized shamcontrolled trial. Clin Neurophysiol 132(1):126-136

25. Grazzi L, Usai S, Bolognini N, Grignani E, Sansone E, Tramacere I et al (2020) No efficacy of transcranial direct current stimulation on chronic migraine with medication overuse: A double blind, randomised clinical trial. Cephalalgia. 40(11):1202-1211 
26. Mansour AG, Ahdab R, Khazen G, El-Khoury C, Sabbouh TM, Salem M et al (2020) Transcranial Direct Current Stimulation of the Occipital Cortex in Medication Overuse Headache: A Pilot Randomized Controlled Cross-Over Study. J Clin Med 9(4)

27. Pohl H, Moisa M, Jung HH, Brenner K, Aschmann J, Riederer F et al (2021) Long-Term Effects of Self-Administered Transcranial Direct Current Stimulation in Episodic Migraine Prevention: Results of a Randomized Controlled Trial. Neuromodulation. 24(5):890-898

28. Rahimi MD, Fadardi JS, Saeidi M, Bigdeli I, Kashiri R (2020) Effectiveness of cathodal tDCS of the primary motor or sensory cortex in migraine: $A$ randomized controlled trial. Brain Stimul 13(3):675-682

29. Rocha S, Melo L, Boudoux C, Foerster A, Araujo D, Monte-Silva K (2015) Transcranial direct current stimulation in the prophylactic treatment of migraine based on interictal visual cortex excitability abnormalities: A pilot randomized controlled trial. J Neurol Sci 349(1-2):33-39

30. Wickmann F, Stephani C, Czesnik D, Klinker F, Timaus C, Chaieb L et al (2015) Prophylactic treatment in menstrual migraine: A proof-of-concept study. J Neurol Sci 354(1-2):103-109

31. Przeklasa-Muszynska A, Kocot-Kepska M, Dobrogowski J, Wiatr M, Mika J (2017) Transcranial direct current stimulation (tDCS) and its influence on analgesics effectiveness in patients suffering from migraine headache. Pharmacol Rep 69(4):714-721

32. Negro A, Martelletti P (2019) Patient selection for migraine preventive treatment with anti-CGRP( $r$ ) monoclonal antibodies. Expert Rev Neurother 19(8):769-776

33. Tiseo C, Ornello R, Pistoia F, Sacco S (2019) How to integrate monoclonal antibodies targeting the calcitonin gene-related peptide or its receptor in daily clinical practice. J Headache Pain 20(1):49

34. Ambrosini A, Schoenen J (2006) Electrophysiological response patterns of primary sensory cortices in migraine. J Headache Pain 7(6):377-388

35. Coppola G, Pierelli F, Schoenen J (2007) Is the cerebral cortex hyperexcitable or hyperresponsive in migraine? Cephalalgia. 27(12):14271439

36. DaSilva AF, Truong DQ, DosSantos MF, Toback RL, Datta A, Bikson M (2015) State-of-art neuroanatomical target analysis of high-definition and conventional tDCS montages used for migraine and pain control. Front Neuroanat 9:89

37. Kurt E, Henssen D, Steegers M, Staal M, Beese U, Maarrawi J et al (2017) Motor Cortex Stimulation in Patients Suffering from Chronic Neuropathic Pain: Summary of Expert Meeting and Premeeting Questionnaire. Combined with Literature Review World Neurosurg 108:254-263

38. Lorenz J, Minoshima S, Casey KL (2003) Keeping pain out of mind: the role of the dorsolateral prefrontal cortex in pain modulation. Brain. 126(Pt 5): 1079-1091

39. Aurora SK, Welch KM, Al-Sayed F (2003) The threshold for phosphenes is lower in migraine. Cephalalgia. 23(4):258-263

40. Vigano A, D'Elia TS, Sava SL, Auve M, De Pasqua V, Colosimo A et al (2013) Transcranial Direct Current Stimulation (tDCS) of the visual cortex: a proofof-concept study based on interictal electrophysiological abnormalities in migraine. J Headache Pain 14:23

41. Ayata C (2013) Pearls and pitfalls in experimental models of spreading depression. Cephalalgia. 33(8):604-613

42. Charles A, Brennan K (2009) Cortical spreading depression-new insights and persistent questions. Cephalalgia. 29(10):1115-1124

43. Kramer DR, Fujii T, Ohiorhenuan I, Liu CY (2016) Cortical spreading depolarization: Pathophysiology, implications, and future directions. J Clin Neurosci 24:22-27

44. Hadjikhani N, Sanchez Del Rio M, Wu O, Schwartz D, Bakker D, Fischl B et al (2001) Mechanisms of migraine aura revealed by functional MRI in human visual cortex. Proc Natl Acad Sci U S A 98(8):4687-4692

45. Lauritzen M (1994) Pathophysiology of the migraine aura. The spreading depression theory Brain 117(Pt 1):199-210

46. Burstein R, Noseda R, Borsook D (2015) Migraine: multiple processes, complex pathophysiology. J Neurosci 35(17):6619-6629

47. Clarke BM, Upton AR, Kamath MV, Al-Harbi T, Castellanos CM (2006) Transcranial magnetic stimulation for migraine: clinical effects. J Headache Pain 7(5):341-346

48. Hansen JM, Charles A (2019) Differences in treatment response between migraine with aura and migraine without aura: lessons from clinical practice and RCTs. J Headache Pain 20(1):96

\section{Publisher's Note}

Springer Nature remains neutral with regard to jurisdictional claims in published maps and institutional affiliations.

\section{Ready to submit your research? Choose BMC and benefit from:}

- fast, convenient online submission

- thorough peer review by experienced researchers in your field

- rapid publication on acceptance

- support for research data, including large and complex data types

- gold Open Access which fosters wider collaboration and increased citations

- maximum visibility for your research: over $100 \mathrm{M}$ website views per year

At BMC, research is always in progress.

Learn more biomedcentral.com/submissions 